\title{
Diseño de Sistema de Innovación Local (SIL) para el Município Camajuaní, Cuba
}

\author{
Hernández Medina Carlos Alberto ${ }^{1 *}$, Báez Hernández Alexander ${ }^{2}$ y Carrasco Fuentes Magdalys Alibet ${ }^{3}$
}

\begin{abstract}
'Profesor Auxiliar. Centro Universitario Municipal Camajuaní. Universidad Central "Marta Abreu" de Las Villas.
${ }^{2}$ Profesor. Universidad Central de Ecuador. Avenida de las Américas. Quito. Ecuador.

${ }^{3}$ Profesor Auxiliar. Centro Universitario Municipal Camajuaní. Universidad Central "Marta Abreu" de Las Villas.
\end{abstract}

\section{Resumen}

El trabajo contiene una conceptualización de los SIL en diferentes áreas geográficas. Está dirigido a la solución de los problemas que necesitan la aplicación del conocimiento para resolverse en el Municipio Camajuaní. Define la importancia e impacto de un SIL sobre la vida del Territorio y que esta depende de variados factores. Se recomienda extender esta experiencia a todas las formas productivas de la municipalidad, capacitar a los actores del contexto comunitario y lograr del gobierno su papel orientador, coordinador y controlador del proceso de intervención comunitaria y desarrollo sociocultural. Se hace una propuesta de estructura institucional para fortalecer el SIL.

Palabras Clave: Sistemas de Innovación Local, SIL, conceptualización, intervención comunitaria, desarrollo local.

\section{Design of Local Innovation System for Camajuaní Municipality, Cuba}

\begin{abstract}
The work contains a conceptualization of SLI in different geographical areas. It is aimed at solving the problems that need the application of knowledge to be resolved in the Camajuaní Municipality. It defines the importance and impact of a SLI on the life of the Territory and that it depends on various factors. It is recommended to extend this experience to all productive forms of the municipality, to train actors of the community context and to achieve of the government its role of counselor, coordinator and controller of the process of community intervention and sociocultural development. A proposal of institutional structure is made to strengthen the LIS.
\end{abstract}

Keywords: Local Innovation Systems, LIS, conceptualization, community intervention, local development. 


\section{INTRODUCCION}

La dinámica de generación y uso de la tecnología es un aspecto crítico que determina el desarrollo de países y territorios y, por tanto, en la competitividad de las empresas. Este problema está centrado, evidentemente, en la capacidad del territorio y sus empresas de generar innovación tecnológica. Por ello, desarrollar condiciones que fortalezcan la capacidad de innovar es un punto que territorios, empresas y gobiernos deben priorizar como elemento esencial de su política y estrategia a mediano y largo plazo. (Terneus et al., 2002)

La necesidad de incrementar el conocimiento tecnológico promoviendo la innovación surge por la creciente importancia del empleo del conocimiento y prácticas tecnológicas en función de la competitividad y porque muchos conocimientos tecnológicos se desarrollan, maduran y obsolecen en corto tiempo. La introducción de innovaciones al sector productivo de bienes y servicios es un proceso complejo en que interactúan numerosos actores:

- Los investigadores y científicos generan nuevo conocimiento y capacitan al recurso humano

- Los empresarios introducen los conocimientos y desarrollos a la producción y la sociedad.

- El sector financiero ofrece alternativas para los costos y riesgos económicos de todo desarrollo.

- El gobierno norma la interacción intersectorial para la innovación y orienta estratégicamente la Investigación, Desarrollo e Innovación; sosteniéndola con fondos públicos.

En un tema tan complejo y que involucra grupos y sectores sociales con objetivos y puntos de vista tan disímiles, se producen conflictos de intereses, dificultades de comunicación y estancamientos que deben ser superados. Para ello es necesario desarrollar un sistema de contactos entre sectores, experiencias comunes y proyectos conjuntos. Estos fortalecen la confianza mutua construyendo formas de coordinación y cooperación, muchas veces informales, que potencian las capacidades en cada sector y facilitan el desarrollo y puesta en marcha de un Sistema de Innovación Local.

A la hora de definir las características de un Sistema de Innovación Local un punto esencial para evitar interpretaciones erróneas es que este debe entenderse como un campo abierto diseñado para facilitar la interacción entre los actores locales (Hernández et al., 2015). En el mismo cada actor cumple su rol realimentando y modificando la percepción que él tiene del problema y ajustándola a la de los demás. Por tanto, este concepto necesita de un comportamiento diametralmente opuesto al de una dirección vertical y demasiado centralizada que se basa en establecer metas a alcanzar y plazos.

A diferencia de lo acostumbrado, un SIL trabaja en la búsqueda de consensos y en la identificación de los objetivos que son comunes a los distintos actores locales. Esta búsqueda se debe realizar desde la diversidad de puntos de vista de cada actor. En un Sistema de Innovación Local consolidado los caminos de vinculación informales son sumamente importantes $y$, a través de ellos se pueden construir las bases reales para el consenso (Hernández et al., 2018). La dinámica de este proceso es impulsada por los propios resultados de la innovación y el marco normativo y legal debe acompañarla. Se deben encontrar mecanismos que permitan su ágil actualización reduciendo al mínimo la burocracia.

La importancia e impacto de un Sistema de Innovación Local sobre la vida del Territorio depende de estar asociado a la demanda, en tanto debe vincularse a las necesidades de los actores económicos locales y estar vinculado a la oferta $y$, por tanto, relacionado con la capacidad de generar innovación tecnológica viable en respuesta a estas necesidades (Hernández, 2015). En relación con esto definiremos algunos conceptos importantes para caracterizar el Sistema de Innovación Local de un Territorio.

Una innovación tendrá éxito en la medida que tome en cuenta no sólo el aspecto tecnológico, sino la estrategia de introducción, manejando el financiamiento, la comercialización y la resistencia al cambio.

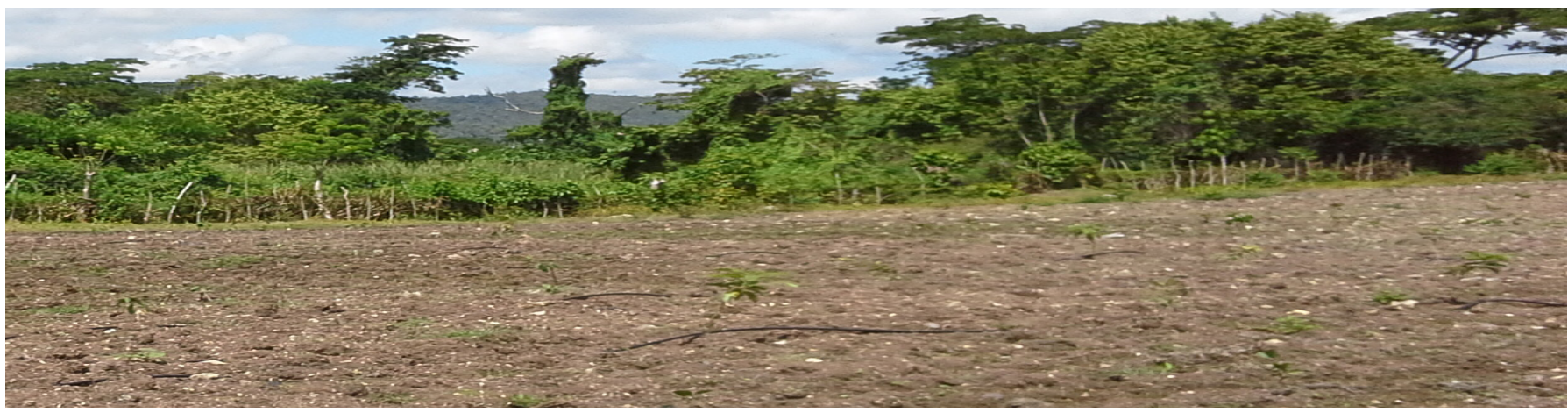


Según Nelson (1993) el tipo de innovación a introducir determina 3 situaciones: 1. Desarrollo de productos y servicios que sean novedades absolutas a nivel mundial dirigidos al mercado internacional (Innovación pionera), 2. Introducción de productos novedosos a nivel mundial, pero dirigidos a mercados existentes, para los que hay competencia internacional (Innovación profunda) y 3 . Aprovechamiento de ventajas locales para desarrollar productos ya existentes, con miras a una competencia que normalmente está basada en la sustitución de importaciones, aunque puede llevar a un mercado más amplio (Innovación adaptativa, local o internacional)

Para poder llevar adelante un análisis de las características de los Sistemas de Innovación Local en Cuba es necesario recordar que, desde fines del siglo XIX, y hasta hace no mucho tiempo, la sociedad cubana percibía la investigación científica, de modo casi unánime, como una más de las actividades culturales del país. Sólo en las últimas décadas, y ante la imposibilidad de continuar sosteniendo la economía nacional a partir de la renta generada por la producción cañera, algunos sectores comenzaron a reconocer el significado económico que tienen las tareas de investigación y desarrollo (Báez et al., 2018).

Por esta razón, en este trabajo expondremos algunos datos concretos referidos a la acción de los actores de los Sistemas de Innovación Local que consideramos significativos. A partir de ellos se obtendrán conclusiones que pretenden constituir una contribución para orientar las acciones futuras.

\section{DESARROLLO}

\section{Ciencia, tecnología e innovación vs. desarrollo:}

Los resultados de Bértola et al. (2006), en una investigación sobre la situación de la ciencia, tecnología e innovación en Uruguay desarrollada por un equipo de investigadores de la Universidad de la República, nos ponen al tanto de que desde que los gobiernos y los organismos internacionales de cooperación reconocieron la importancia de promover la ciencia y la tecnología como elementos fundamentales para la evolución de la economía han aparecido varios enfoques para el análisis e implementación de estrategias de ciencia y tecnología.

Durante algunas décadas, predominaron diversas variantes del concepto de Sistema Nacional de Ciencia y Tecnología, asociado a un modelo lineal del proceso de innovación que concentra los esfuerzos en apoyar la producción de nuevos conocimientos y tecnologías en universidades, centros de investigación y departamentos de I+D de las empresas. Este conocimiento, codificado, luego desencadenaría un proceso que terminaría generando nuevos productos y procesos.

Los magros resultados de las políticas basadas en estos modelos, así como los estudios empíricos de experiencias exitosas en algunos países, llevaron a desarrollar el concepto de SNI como forma de expresar el carácter interactivo y dinámico del proceso innovador. Probaron también su condición de proceso de aprendizaje social, acumulativo y

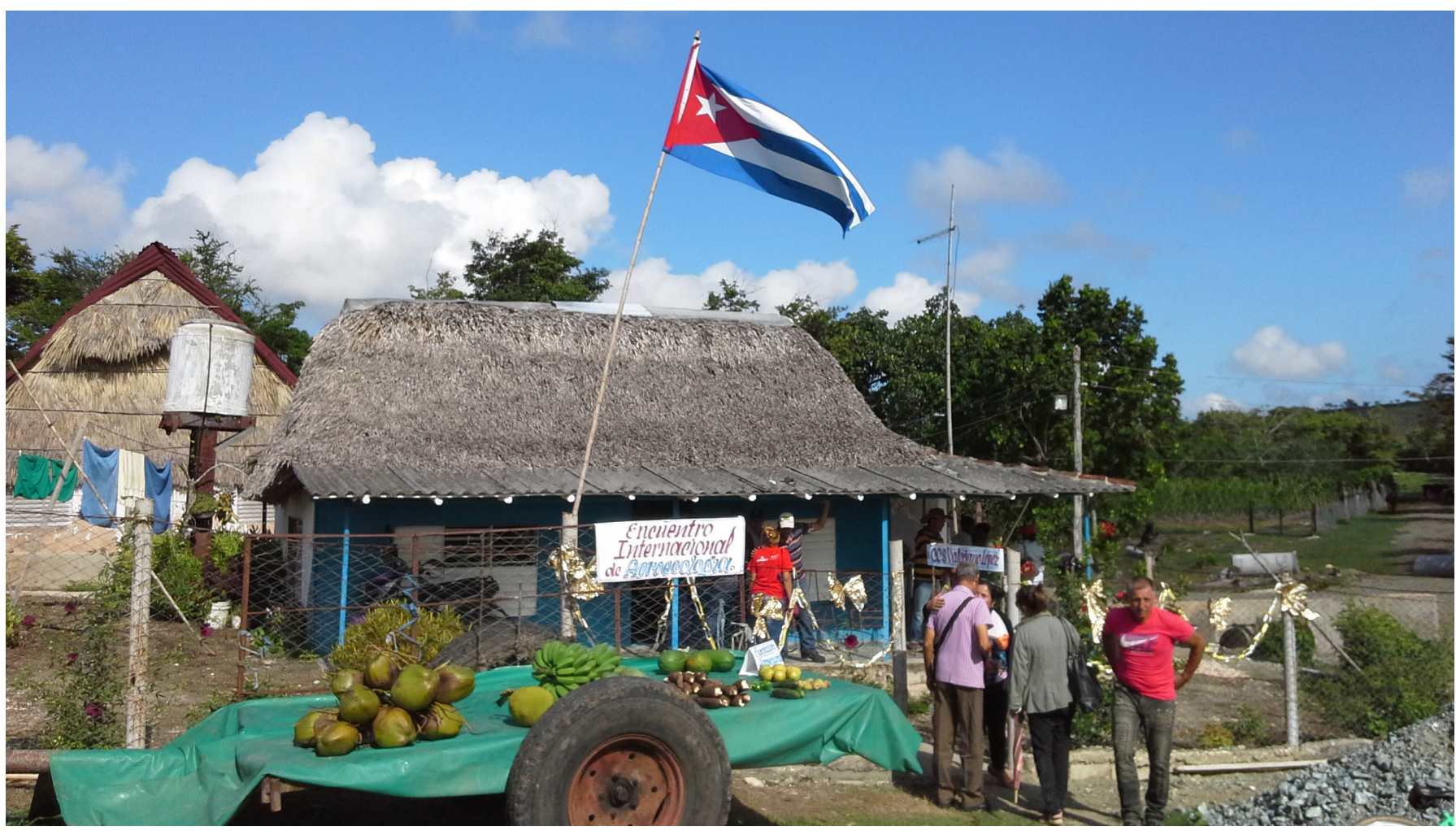




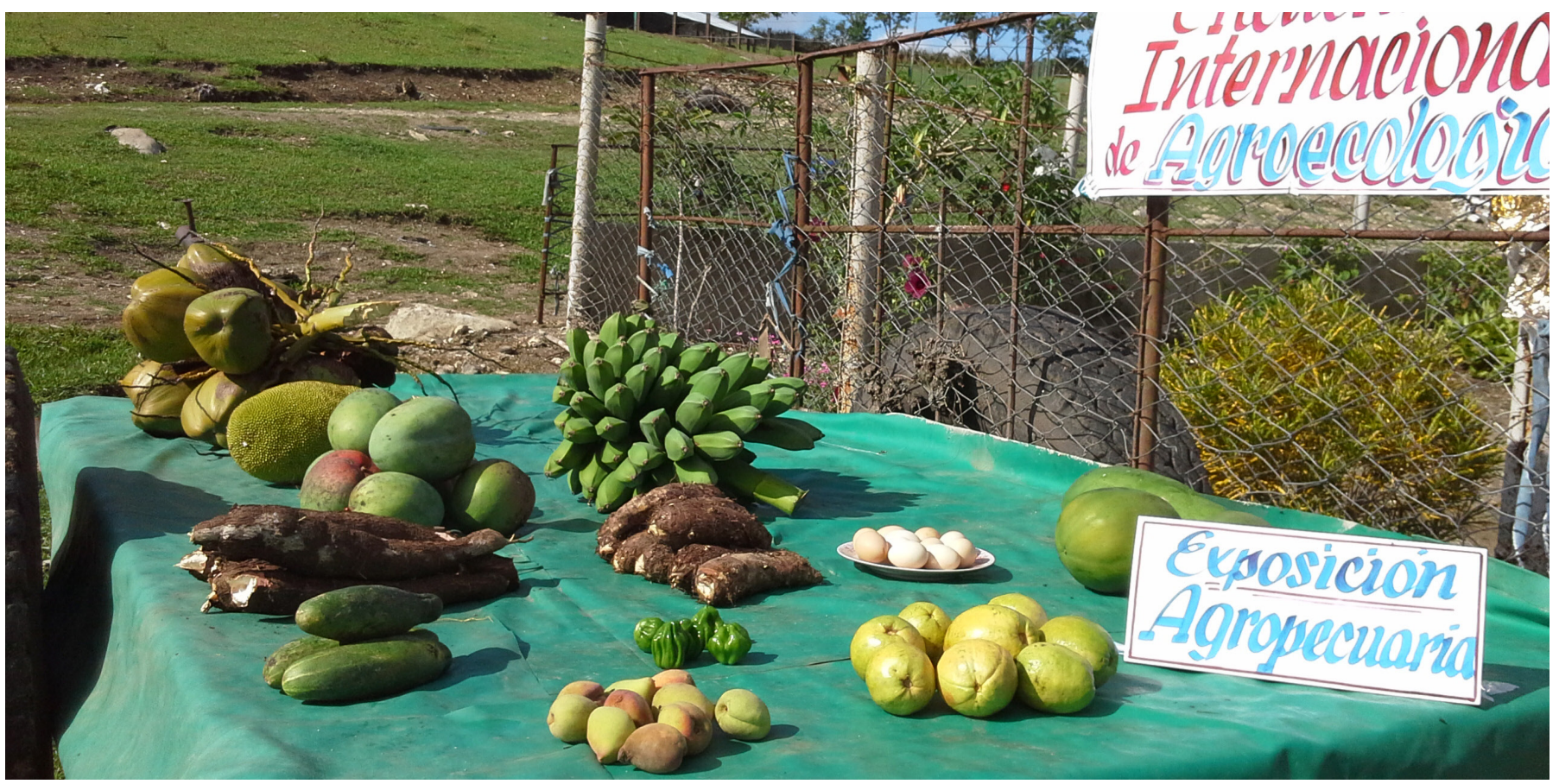

la importancia del conocimiento tácito en su conducción. Este novedoso concepto pretende capturar la idea de que el cambio tecnológico es un fenómeno sistémico que va más allá de las esferas de la ciencia y la tecnología en sentido estricto. Comprende desde la generación del nuevo conocimiento hasta su aplicación y su difusión al conjunto de la economía. (Bértola et al., 2006)

\section{Concepto de Sistema Nacional de Innovación (SNI)}

El concepto de SNI fue introducido por Freeman, Lundvall y Nelson quienes, desde diferentes perspectivas, estudiaron los mecanismos de generación y difusión del conocimiento en países desarrollados. Coinciden en subrayar el papel de la empresa como depositaria principal del conocimiento tecnológico y el de las instituciones y mecanismos que influyen sobre la dirección e intensidad del cambio técnico. Además, comparten la visión de que la innovación tecnológica, vista como producto de la ciencia aplicada en la praxis social, es un proceso no lineal, cuyo desempeño depende estrechamente de la existencia de estructuras organizativas que posibiliten las interacciones entre los distintos agentes sociales en juego en la localidad.

Para ellos la tecnología es, en su mayor parte, resultado de la acumulación de conocimientos por parte de las empresas. Argumentan que las firmas van acumulando el conocimiento mediante un proceso de aprendizaje interno a la empresa y justifican que lo van adquiriendo a lo largo de una trayectoria tecnológica propia. Es este el resultado de admitir que el progreso técnico es endógeno a las empresas y que éstas son su agente protagónico. Aseguran también que ese proceso es potenciado u obstaculizado por el entorno productivo inmediato a la empresa, formado por redes con otras firmas del mismo y de otros sectores de la actividad económica local. El tipo de trama productiva existente y los conocimientos que fluyen por ellas definirán las características básicas del SNI del país.

Un tercer componente del SNI está compuesto por las entidades de la infraestructura tecnológica como son las universidades y agentes públicos que generan conocimiento, las interconexiones entre ellas y sus relaciones con las empresas que innovan. También influyen en el proceso de innovación las formas de organización, las convenciones y los comportamientos prevalecientes en la empresa, en su entorno y en la economía en general. En resumen, una manera de conocer el SNI de un país consiste en analizar el comportamiento innovativo de las empresas, las tramas productivas existentes, los conocimientos que fluyen por ellas y la configuración institucional del cambio técnico.

La aplicación a una economía subdesarrollada del concepto de SNI ha de realizarse con suma cautela. Como señalan Arocena y Sutz (2014), el SNI se originó en los países desarrollados como un concepto ex post mientras que en realidades como las nuestras es un concepto ex ante. En el mundo desarrollado el análisis de los SNI ha ayudado a entender las distintas trayectorias y modalidades existentes. En los países en desarrollo este concepto ha sido utilizado principalmente como un modelo que permite detectar metódicamente las debilidades y obstáculos que impiden un mejor desempeño innovador, que, por su mismo carácter sistémico, tienen un profundo impacto en la competitividad del país y en la calidad de vida de la sociedad. 


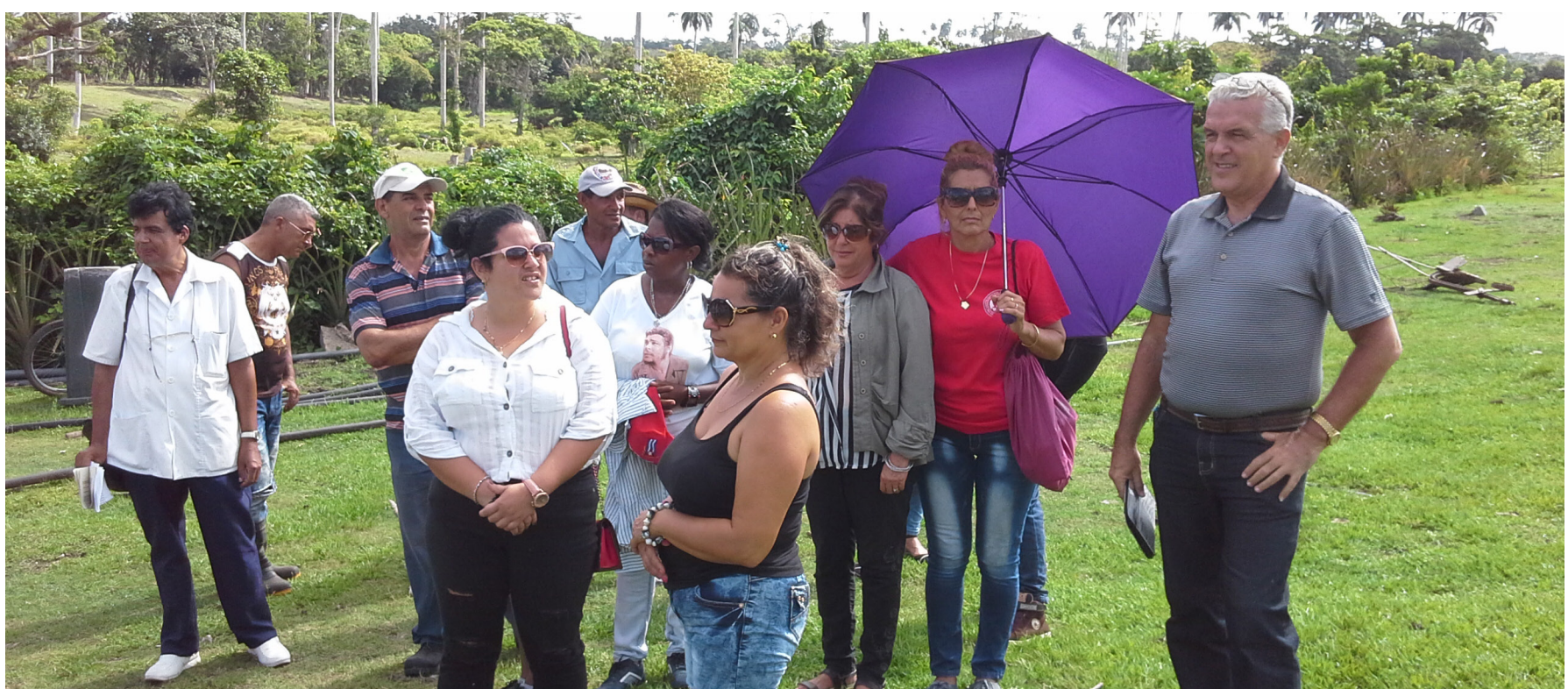

Ver al proceso de innovación como sistema supone que ella penetra al conjunto de las actividades sociales. Lo hace tanto con la producción, transferencia, adopción y consumo de bienes, servicios y conocimiento, como con las diferentes organizaciones vinculadas como son las empresas, dependencias estatales y comunales, organizaciones no gubernamentales, sistemas educativos, hogares y consumidores en general.

Aunque las empresas, los gobiernos y los mercados juegan un papel decisivo, la intensidad y frecuencia del proceso innovador depende de un entorno político, social, cultural y económico más amplio. En él se realiza un variado conjunto de intercambios y procesos no mercantiles, científicos ni productivos que son esenciales. Son las particularidades de estos sistemas integrales los que explican los dispares desempeños económico-sociales entre países, territorios y localidades.

\section{Modos de producción del conocimiento:}

Hace algunos años tuvo una gran repercusión una obra colectiva sobre... "el nuevo modo de producción de conocimiento" escrita por Lundwall y Borrás (2005) referida a una aportación de Gibbons. La idea central planteada por Gibbons era que existe actualmente una transición en las características de las prácticas de investigación desde lo que denominó un "Modo 1" a un "Modo 2" de hacer ciencia.

En el primer modo la investigación tiene lugar en un contexto fundamentalmente académico, se organiza en torno a las disciplinas, se realiza por equipos estables, que trabajan en el "contexto de descubrimiento" en instituciones también estables, respondiendo preguntas que surgen principalmente desde 'el interior' de la ciencia. Sus resultados impactan en la sociedad al ser difundidos por actores diferentes a los investigadores, luego de ser evaluados por ellos mismos al interior de la comunidad científica y de sus propias disciplinas.

En el "Modo 2" la investigación se realiza principalmente orientada a la aplicación, de manera trans-disciplinaria, se organiza a través de equipos a término, trabajando sobre problemas que se plantean desde 'el exterior' de la academia, produciendo resultados en distintas instituciones y lugares que se vinculan de diversas maneras. Sus resultados son evaluados no sólo por los pares científicos, sino respondiendo a un amplio espectro de intereses, e impactan en la sociedad desde el momento de la definición de los problemas y prioridades, en el inicio del proceso de investigación.

En realidad, es muy posible que nunca haya existido un Modo 1 en forma pura, sino alguna combinación, variable según áreas del conocimiento, grado de avance del conocimiento, problemas de la realidad y contextos socio-económicos. También es probable que realmente se asista hoy a una nueva combinación altamente variable de acuerdo al campo de estudios y al problema considerado de ambos modos más que a una transición o contraposición.

Esta discusión planteada por Gibbons et al., (1997) tiene cierta relación, aunque no directa, con otro libro de gran repercusión publicado al mismo tiempo por Ziman (2003). En el planteaba, entre otras cosas, los cambios en proceso, a fines del siglo $X X$, en las prácticas de la investigación de científicos y tecnólogos. En ellas se observa, según el autor, un paso del comportamiento según las clásicas normas mertonianas a formas nuevas, basadas en nuevos principios. El paso de CUDOS (Comunalismo, Universalismo, Desinterés, 
Originalidad y Escepticismo) a PLACE (Propiedad, Local, Autoritario, Comisionado, trabajo Experto).

Estas discusiones conceptuales pueden parecer arbitrarias al realizar propuestas de políticas científico-tecnológicas. Sin embargo, son importantes para mostrar que el mayor desafío para nuestros países es la imprescindible transformación de la valoración social de la Ciencia e Innovación Tecnológica. Lo debemos hacer complementando la visión predominante, orientada a "descubrir", con una visión orientada a "resolver problemas". Esto la vinculará no sólo con la cultura sino también con la economía y el desarrollo del país. Debemos hacer énfasis no sólo en la generación de conocimiento nuevo sino en su uso y aplicación en la solución de los problemas locales. Es imperativo situar a la Ciencia e Innovación Tecnológica como un área ineludible a la hora de considerar cualquier estrategia sustentable de desarrollo nacional y local.

\section{Para aplicar estos conceptos hay afirmaciones que se derivan de ellos:}

Las capacidades de innovación son fuente de ventaja competitiva: El objetivo de las políticas de Ciencia e Innovación Tecnológica (CeIT) es contribuir a la expansión de las capacidades innovadoras y creativas de la sociedad. Sobre ellas se apoya, en altísimo grado, el desarrollo de la localidad y el país. Esas capacidades, canalizadas a través de un eficiente sistema de estímulos, que abarca actores públicos y privados, en un contexto de una dinámica inserción regional e internacional, deben traducirse en mejoras de la capacidad competitiva tanto de las empresas individuales como del entramado social y productivo local considerado como sistema.

La competitividad debe ser el sustento de un desarrollo equitativo: La expansión de las capacidades innovadoras, como base de la competitividad internacional y del modelo de crecimiento, no puede estar desligada, por razones éticas y de eficiencia, de formas justas de distribución de la riqueza y del poder político, social y económico. La competitividad debe ser sustento de desarrollo local integrador y equitativo, democrático y transparente.

Se necesitan reglas claras y estables del entorno institucional: La existencia de entornos institucionales favorables a la innovación es la clave del desarrollo local integral, económico y social. La innovación como proceso sistémico necesita de una cultura apropiada y de entornos institucionales que la promuevan. La innovación no es resultado de actos voluntaristas ni de construcciones institucionales impuestas, poco relacionadas con la práctica cotidiana y de difícil aplicación y seguimiento. Es un proceso lento, acumulativo, con muchos componentes rutinarios. Necesita también la existencia de amplio consenso político y social que asegure la persistencia de las políticas, a pesar de movimientos políticos circunstanciales.

Las políticas de innovación como pilar del desarrollo tienen un componente transversal: Una consecuencia del enfoque

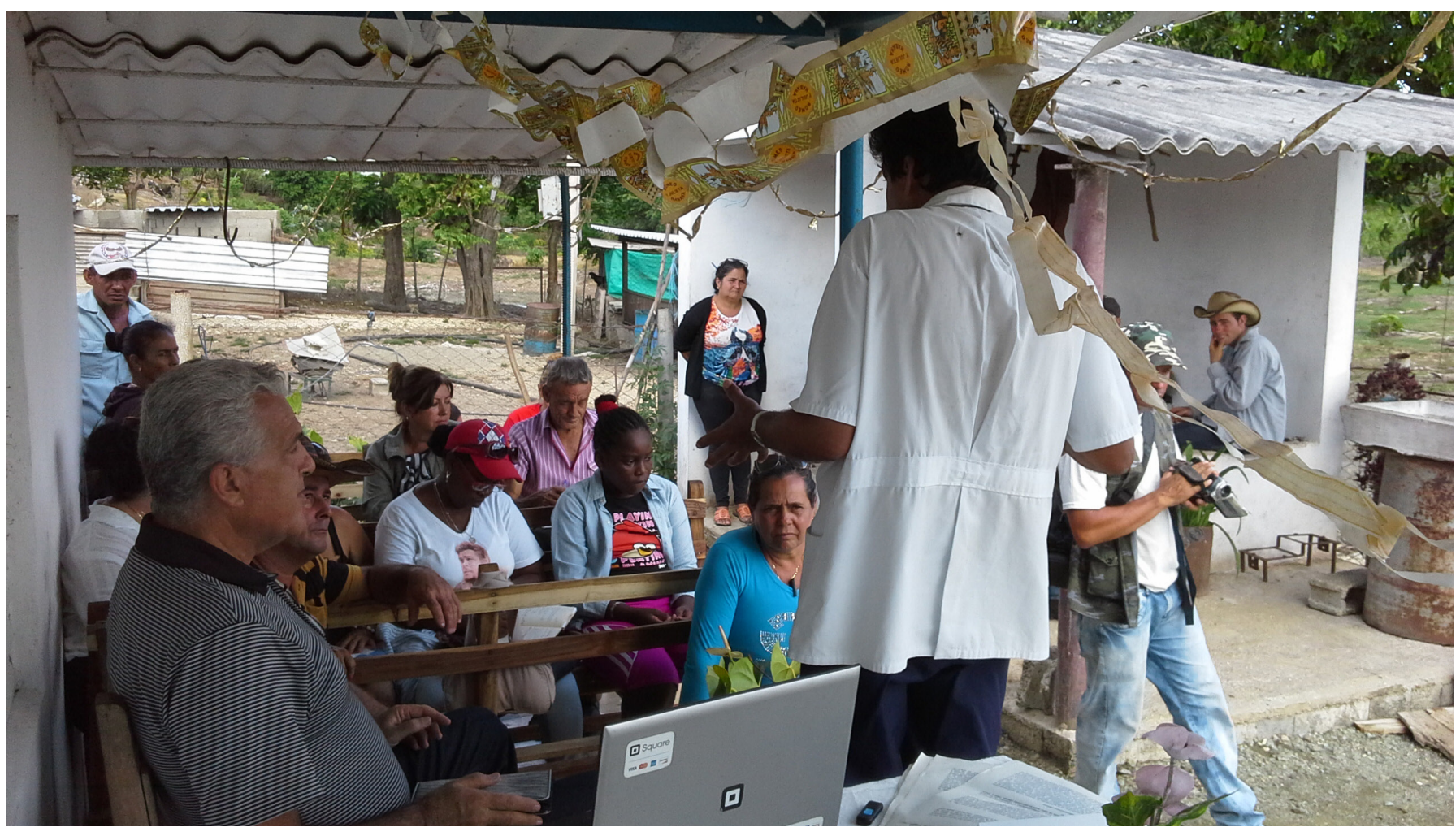


sistémico es que existen múltiples áreas o dimensiones de política que tienen una incidencia directa sobre las capacidades innovadoras de una sociedad. La creación de un ambiente amigable a la innovación sólo es posible con políticas coordinadas en áreas como educación, investigación, producción, tributaria y financiera. Las políticas transversales buscan evitar la fragmentación de la capacidad innovadora, la creación de dualismos y la subestimación de capacidades de diferentes actores llegando al conjunto del tejido socio-económico. Constituyen una importante herramienta para contrarrestar particularismos y conductas buscadoras de ganancias a toda costa.

Las políticas específicas deben ser complemento de las transversales: Junto a los instrumentos horizontales de política que apoyen proyectos o ideas innovadoras, independientemente del sector económico o área del conocimiento de que provengan, es necesario desarrollar políticas específicas: sectoriales, locales, empresariales, de tramas productivas o polos de desarrollo, que partan de una identificación de áreas estratégicas del conocimiento y de la economía. Dichas políticas sectoriales deberán aplicar recursos y capacidades específicas, ya sea por su impacto social (empleo, ingreso, género), su capacidad de penetración exportadora, su importancia en la frontera de la ciencia o por su impacto local.

La investigación científica y tecnológica debe ser un elemento estratégico: Un componente clave del Sistema de Innovación Local es la investigación científica y tecnológica y la formación de investigadores. Aunque toda investigación científica no ha de tener un impacto directo en la innovación, el desarrollo de capacidades científicas es un componente clave de una cultura nacional de innovación y transformación de la sociedad.

La alfabetización científico-tecnológica de la población ha sido siempre en Cuba una meta central desde el triunfo de la Revolución en 1959. La innovación se basa en el conocimiento científico. La capacidad de desarrollar un sistema de innovación supone la existencia de fuertes núcleos de producción científica. La existencia de un pensamiento científico independiente y con criterios propios de evaluación, fundamentalmente orientado a la solución de problemas específicos, constituye una parte indispensable de ese sistema. Particularmente importante es la investigación en ciencias sociales dada la especificidad histórica y geográfica de las conformaciones sociales que obliga a combinar la búsqueda de patrones universales con una fuerte de investigación particular.

Un sistema de innovación sólo lo es en la medida que existen fuertes interacciones entre los diversos actores. Son los vínculos los que determinan la concepción sistémica. Esas interacciones pueden tomar la forma de competencia, transacciones o redes colaborativas. Operan bajo diversas estructuras de mercado y otras relaciones sociales.

Los mecanismos de estímulo son decisivos, aunque pueden configurarse de manera perversa. Si quienes hegemonizan su estructura explotan rentas de privilegio, es necesario defender las rentas de innovación de manera dinámica, ya que las estructuras de mercado no son generadoras de procesos de aprendizaje. Procesos de liberalización comercial pueden dejar expuestos a sectores estratégicos de la economía desde el punto de vista de la dinámica científico-tecnológica y el potencial de contribución a los componentes sistémicos de la innovación.

Al interior de esta corriente han existido diferentes momentos de las políticas científico-tecnológicas aplicadas en las últimas décadas en Latinoamérica. Algunas versiones tempranas ponían énfasis en los aspectos formales de los sistemas y enfatizaban el rol jugado por el Estado en la construcción de las infraestructuras científicotecnológicas. Esperaban que los resultados penetraran a la sociedad, generando innovación y desarrollo. Tendencias posteriores han enfatizado el rol de la innovación a nivel de las empresas, vista la escasa vinculación lograda entre los esfuerzos científicos y la producción. Las políticas transversales orientadas a encontrar a los innovadores donde se encuentren no han logrado aún revertir el problema de que la demanda por ciencia y tecnología para la innovación resulta muy escasa.

Las teorías de los SNI vuelven a hacerse las viejas preguntas de las diferentes corrientes sobre cómo atacar los problemas idiosincrásicos y culturales que no valoran la importancia de la innovación, cómo revertir las especializaciones productivas que nos atan a los recursos naturales, cómo atacar las conductas buscadoras de rentas de privilegio. Buscan cómo convertir al Estado en una fuente de dinamismo; cómo transformar la idea de que las estructuras de mercado son las idóneas y cómo lograr la cooperación y la interacción entre los diversos agentes del sistema.

\section{Problemáticas y desafíos del SNI cubano}

Se articulan en torno a tres ejes. El primero es la "Cultura Innovadora": El modelo de especialización productiva en industrias de bajo valor agregado y potencialidades tecnológicas reducidas y la inhibición del desarrollo de capacidades tecnológicas domésticas atenta contra el funcionamiento de tramas productivas locales que facilitan la internalización de las economías externas derivadas del cambio tecnológico. Aspectos históricos, económicos, sociales, políticos y culturales contribuyen a una cultura empresarial escasamente propensa a la innovación. Las entidades dedicadas a la Ciencia e Innovación Tecnológica 
han desarrollado hábitos de comportamiento y estilos de trabajo burocratizados que han tornado difícil la transferencia de tecnología hacia el aparato productivo.

Desafío: La transformación de las actitudes sociales hacia la innovación abarca principalmente la generación de estímulo y reconocimiento hacia el empresariado y los dirigentes sobre la importancia de la capacidad de las organizaciones de agregar valor a través del conocimiento, diversificando radicalmente la estructura productiva; la formación de la ciudadanía en temas de CelT para la incorporación de estas dimensiones a la cultura ciudadana.

Este cambio cultural debe expresarse en un incremento sustancial de la inversión en I+D. Se debe incentivar el surgimiento de nuevas empresas de base tecnológica y promover a nivel empresarial la innovación tecnológica como herramienta clave para el incremento de la productividad. El interés que despierta en la dirigencia política el fenómeno de la innovación debe plasmarse en un plan estratégico nacional y local. El Estado debe promover el estudio sobre el desarrollo con capacidad de prospectiva en materia de CelT.

Interacción entre los Actores del Sistema: Al débil entramado empresarial señalado en el punto anterior como causa de una escasa interacción y articulación de la base productiva nacional, se suma un conjunto de entidades que participan en el proceso de cambio tecnológico de una forma "espontánea". Este modelo puede resultar funcional en algunas sociedades, pero en una sociedad empresarialmente débil como la nuestra, el conjunto de entidades existente abarca muchas funciones del sistema de Ciencia e Innovación Tecnológica, pero carece de organización interna con competencias suficientes para imponer un plan estratégico en la materia y de una política general explícitamente formulada. Ello resulta en que carezcamos de un "sistema" de innovación a lo interno, ya que los diferentes componentes no funcionan con las interacciones y articulaciones propias de lo que en la literatura teórica se denomina como sistema.

Desafío: Es necesario crear una estructura institucional adecuada a las características del país y de las políticas que se han de llevar a cabo, que considere al carácter transversal del fenómeno de la innovación y a la vez sea flexible para permitir tanto el necesario proceso de aprendizaje que el país debe recorrer, como su articulación con políticas sectoriales específicas. La coordinación de las políticas es esencial para evitar superposiciones, atomización y derroche de recursos. La estructura institucional deberá insertarse al más alto nivel y permitir una adecuada articulación con las metas más generales de una política de desarrollo. La política hacia las empresas debe contemplar la gran diversidad existente entre las mismas y explorar diferentes formas de relacionarse (competitivas, de transacciones o de colaboración) entre ellas y otros actores del sistema. Se propone un programa de extensionismo tecnológico orientado a empresas que están más alejadas de la cultura de la innovación, por lo que requieren de otro tipo de apoyos. También en este ámbito se explora posibles políticas a seguir para promover efectos positivos a partir de la interacción de las empresas nacionales con las extranjeras.

\section{RESULTADOS Y DISCUSION}

\section{Estructura institucional para fortalecer el SNI.}

Esta sección se orienta a mostrar una estructura institucional que organiza a uno de los actores del sistema, el Estado, de manera que logre fortalecer su acción sistémica.

Puede decirse que la toma de decisiones y la ejecución de acciones en materia de Ciencia e Innovación Tecnológica se produce en cuatro niveles:

a) Nivel Estratégico y de Definición de Políticas

b) Nivel de Coordinación y Apoyo de Políticas Específicas

c) Nivel de Gestión de Programas

d) Nivel de Ejecución de actividades de I+D e Innovación

Laformaylosámbitosdondeocurreel diseñoyejecución de políticas en el último nivel merecen una fuerte transformación. Actualmente existen instituciones que operan a nivel estratégico y de definición de políticas. Asimismo, a nivel de coordinación y apoyo de políticas específicas encontramos al CITMA que está involucrado a nivel de gestión de programas, con una Unidad de Coordinación a nivel local que cumple esta tarea.

Aunque hemos optado por seguir el orden lineal de los niveles expuesto anteriormente, es necesario resaltar el carácter central que juega el Ministerio de Ciencia, Tecnología y Medio Ambiente (CITMA), ubicado en cada nivel, en el diseño y ejecución de políticas nacionales en la materia.

\section{CONCLUSIONES}

1. Los territorios, gobiernos y empresas deben priorizar como elemento esencial de su política y estrategia a mediano y largo plazo el fortalecer su capacidad de realizar innovación tecnológica.

2. La importancia e impacto de un Sistema de Innovación 
Local sobre la vida del Territorio depende de su vinculación a las necesidades de los actores locales y de su capacidad de generar innovaciones tecnológicas viables en respuesta a estas necesidades.

3. El mayor desafío de la ciencia es complementar su tendencia a investigar, con una visión orientada a resolver problemas sociales, vinculándose con la economía y el desarrollo, generando conocimientos y aplicándolos en la solución de los problemas del país.

4. La innovación como sistema penetra al conjunto de las actividades sociales (producción, transferencia, adopción y consumo de bienes, servicios y conocimiento) y a todas las organizaciones vinculadas.

5. Es necesario crear un Sistema de Innovación adecuado a las características del Territorio y a las políticas que se han de llevar a cabo, que considere al carácter transversal de la innovación y sea flexible para permitir su articulación con las políticas sectoriales. $\mathbf{Q}$,

\section{BIBLIOGRAFÍA}

Arocena, R y Sutz, J. 2014. Mirando los Sistemas Nacionales de Innovación desde el Sur. https://eva.fcs. edu.uy/pluginfile.php/59116/mod_folder/content/0/ Bibliograf\%C3\%ADa\%20obligatoria/u4\%20-\%20 Arocena-Sutz\%202001.pdf?forcedownload $=1$

Báez, A.; Hernández,C.; Perdomo,J. M.; Garcés, R.; Carrasco; M. A. 2018. Modelo de gestión del conocimiento para el desarrollo agropecuario local. Revista Estudios Sociales. No. 51, Vol. 28. pp.: 2 - 26. Ene. - Jun. DOI: https:// dx.doi.org/10.24836/es.v28i51.517

Gibbons, M.; Limoges, C.; Nowotny, H.; Schwartzman, S.; Scott, P.; Trow, M. 1997. La nueva producción del conocimiento. La dinámica de la ciencia y la investigación en las sociedades contemporáneas. Ediciones Pomares Corredor S.A., Barcelona.
Hernández, C. 2015. Relación Universidad Sociedad en función del desarrollo. Lámpsakos. 14. pp. 10-12, Julio-Dic. http:// www.funlam.edu.co/revistas/index.php/lampsakos/article/ view/1706/1421

Hernández, C.; Carrasco, M.; Garcés, R. 2015. Collaborations and links of Municipal University Centre Local Networks of Knowledge and Innovation: Case Study of Camajuaní, Cuba. Proceedings. GlOBELICs 2015. Habana, Cuba. 23 - 25 sept.

Hernández, C.; Garcés, R.; Perdomo, J. M.; Carrasco, M. 2018. Gestión universitaria del conocimiento para el desarrollo local. Lámpsakos. No.18. pp. 44-57. julio-dic. 2017. DOI: http://dx.doi.org/10.21501/21454086.2317

Lundwall, B. A. y Borrás, S. 2005. Política de Ciencia, Tecnología e Innovación. En: Fagerberg, J.; Mowery, D. C. y Nelson, R. 2005 (Eds) Innovation Handbook. Oxford: Oxford University Press. Cap. 22. pp. 599-631.

Nelson, R. 1993. National Systems of Innovation: A Comparative Study. Oxford University Press.

Terneus, A., Marta E. Borda, C. Marschoff. 2002 iExiste un Sistema Nacional de Innovación en Argentina? Sistemas de Innovación. Monográfico. No. 4. Sept-Dic.

Bértola. L., C. Bianchi, P. Darscht, A. Davyt, L. Pittaluga, N. Reig, C. Román, Michele Snoeck, H. Willebald. 2006. Diagnóstico, Prospectiva y Políticas. Consultado el 13 de septiembre de 2009, de: http://www.iadb.org/regions/ re1/econ/RE1-RN-05-001.pdf

Ziman, J. 2003. Ciencia y Sociedad Civil. Revista CTS, No. 1, Vol. 1, Sept. pp. 177-188. ISSN 1668-0030. http:// www.scielo.org.ar/pdf/cts/v1n1/v1n01a10.pdf 